ENCYCLOPEDDIE Encyclopédie berbère

BERBERE

$35 \mid 2013$

35| Oasitae - Ortaïas

\title{
Olivier/Olive : Note linguistique
}

Salem Chaker

\section{OpenEdition}

Journals

Édition électronique

URL : https://journals.openedition.org/encyclopedieberbere/2814

DOI : 10.4000/encyclopedieberbere.2814

ISSN : 2262-7197

\section{Éditeur}

Peeters Publishers

\section{Édition imprimée}

Date de publication : 1 juin 2013

Pagination : $5749-5760$

ISBN : 978-2-7584-0184-1

ISSN : 1015-7344

\section{Référence électronique}

Salem Chaker, «Olivier/Olive : Note linguistique », Encyclopédie berbère [En ligne], 35 | 2013, document 016, mis en ligne le 12 mars 2021, consulté le 17 février 2022. URL : http://journals.openedition.org/ encyclopedieberbere/2814; DOI : https://doi.org/10.4000/encyclopedieberbere.2814

Ce document a été généré automatiquement le 17 février 2022.

(c) Tous droits réservés 


\title{
Olivier/Olive : Note linguistique
}

\author{
Salem Chaker
}

1 [Cette notice a bénéficié des lectures attentives et des suggestions de J. Desanges, J.-V. Vernhes, helléniste de l'Université d'Aix-Marseille, J.-F. Terrai, paléo-botaniste de l'Université de Montpellier-II, M. Thinon, botaniste (CNRS/Université d'Aix-Marseille), M. Tilmatine, de l'Université de Cadix ; nous les en remercions vivement.]

2 La question du nom berbère de l'olivier a déjà été précisément abordée sous les notices "Aliw» et «Azemmur» (“A167", EB IV, 1987 et “A346”, EB VIII, 1990: S. Chaker). L'extension de ce mot azəmmur - «olivier / oléastre » à une très vaste aire du monde berbère - du Djebel Nefoussa* en Libye à l'ensemble du Maroc - et sa grande stabilité formelle (masc. : azəmmur, plur. izəmran ; fém. : tazəmmurt, plur. tizəmrin), déjà relevée par Laoust (1920, p. 447), confirment son caractère très ancien.

Plusieurs compléments et précisions méritent d'être apportés. On ne s'intéressera ici qu'aux termes génériques, à l'exclusion des nombreuses dénominations des diverses variétés cultivées, dont on peut trouver un échantillon kabyle dans Oulebsir (2008).

- On a relevé, au plan sémantique, une distribution fort intéressante: toutes les régions méditerranéennes de l'est et du centre, de la Tripolitaine à la Kabylie, de vieille tradition oléicole, donnent à azəmmur le sens de «olivier (cultivé)»; alors que les régions occidentales ou méridionales (parlers de l'ouest algérien, rifain, tamazight et tachelhit), lui attribuent le sens « oléastre » et empruntent à l'arabe la dénomination de l'olivier cultivé : zzitun, zzutin, zzitunet.

- Conformément au système sémantique de l'opposition de genre en berbère (cf. notices G27 «Genre », EB XX, 1998 et N62 «Nom », EB XXXV, 2012), le masculin (azəmmur) désigne l'espèce et a donc valeur de collectif (= «les oliviers, l'espèce olivier »); alors que le féminin (tazəmmurt), qui est un singulatif, désigne un arbre particulier (= « un olivier, cet olivier »).

- On a signalé que azəmmur présente la forme typique d'un schème de participe passif ${ }^{1}$ (a$\mathrm{C}^{1} \mathrm{C}^{2} \mathrm{C}^{2} \mathrm{uC}^{3}$ ) sur une racine $\mathrm{ZMR}$, qui peut renvoyer à la notion de "pouvoir, supporter, endurer, être capable ». Cette donnée morphologique donne à penser que azəmmur est une forme qualifiante secondaire et non un lexème primitif, et donc que ce n'est pas la dénomination première de l'olivier/oléastre en berbère. Werner Vycichl (1962) à rapproché cette racine berbère ZMR - «pouvoir » du sémitique DMR / ZMR, « force », en considérant 
que la forme berbère remontait à l'héritage chamito-sémitique commun. Pour une vision plus complète du signifié de cette racine, plus complexe que ne le laisse paraitre l'article de W. Vycichl, on se reportera au Dictionnaire des racines sémitiques (Cohen et al., 4, 1993, p. 335-335). Le lien de azəmmur - «Olivier » avec cette racine berbère/chamito-sémitique ZMR est évidemment difficile à établir avec certitude. Néanmoins, s'il était démontré, cela fonderait un lien sémantique entre azəmmur - «Olivier » et la notion de « force, résistance/ endurance, capacité, protection... » qui ne serait pas sans intérêt au plan symbolique quant aux vertus attribuées à l'olivier (voir aussi ci-dessous āliwən). Incidemment cela confirmerait le caractère secondaire de la dénomination azəmmur, évoqué ci-dessus. Bien entendu, ici «secondaire " ne signifie pas « récent » puisque le terme est très largement répandu dans la toponymie nord-africaine et déjà attesté dans les sources médiévales arabes au XI ${ }^{\mathrm{e}}$ siècle (Al-Išbīlī, cf. Bustamente, Corriente et Tilmatine 2007, vol. II, p. 342, nº 2230).

- Le touareg possède une forme āliw, ālew, plur. āliwən pour désigner une variété d'olivier sauvage qui, d'un point de vue botanique (Green 2002 ; Besnard et al. 2009), est une sousespèce distincte de 0 . europaea L. (Olea laperrinei, Batt. \& Trab. ${ }^{2}$; cf. Foucauld, 1951, III, p. 1094 ; Prasse 2003, I, p. 501). Ce terme présente une ressemblance nette - que Charles de Foucauld avait déjà pointée - avec les formes latines olea / oliua/ oleum (olive, olivier, huile

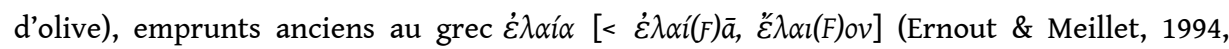
p. 460). Emile Laoust (1920, p. 446-447), évoquant l'importance de la culture de l'olivier pendant la période romaine, notamment en Tripolitaine, admet que le touareg ālew/āliw, a été emprunté au latin, ce qui a une certaine vraisemblance vu l'isolement dialectologique de cette forme et qu'il est établi qu'une partie au moins des actuelles populations touarègues notamment les fameux Huwwara (< Ihəwwarən = Ahaggar / Ihaggaren) - vient de régions libyennes septentrionales, la Tripolitaine (cf. M. Gast, "Huwwara», EB XXIII, 2000 ; Ibn Khaldoûn, I, p. 275-276). A l'appui de cette thèse, Laoust évoque - mais là, il est beaucoup moins convaincant - d'autres possibles emprunts berbères au latin dans le domaine des technologies liées à l'huile d'olive. L'étymologie latine est généralement admise par les auteurs ultérieurs, à l'exception intéressante de l'hispanisant R. Ricard qui, sur la base de la présence d'une forme troublante aléo en portugais, émet de sérieux doutes à son sujet et formule l'idée que : «il s'agirait une fois de plus du fond de vocabulaire commun au monde méditerranéen » (1961, p. 184). 

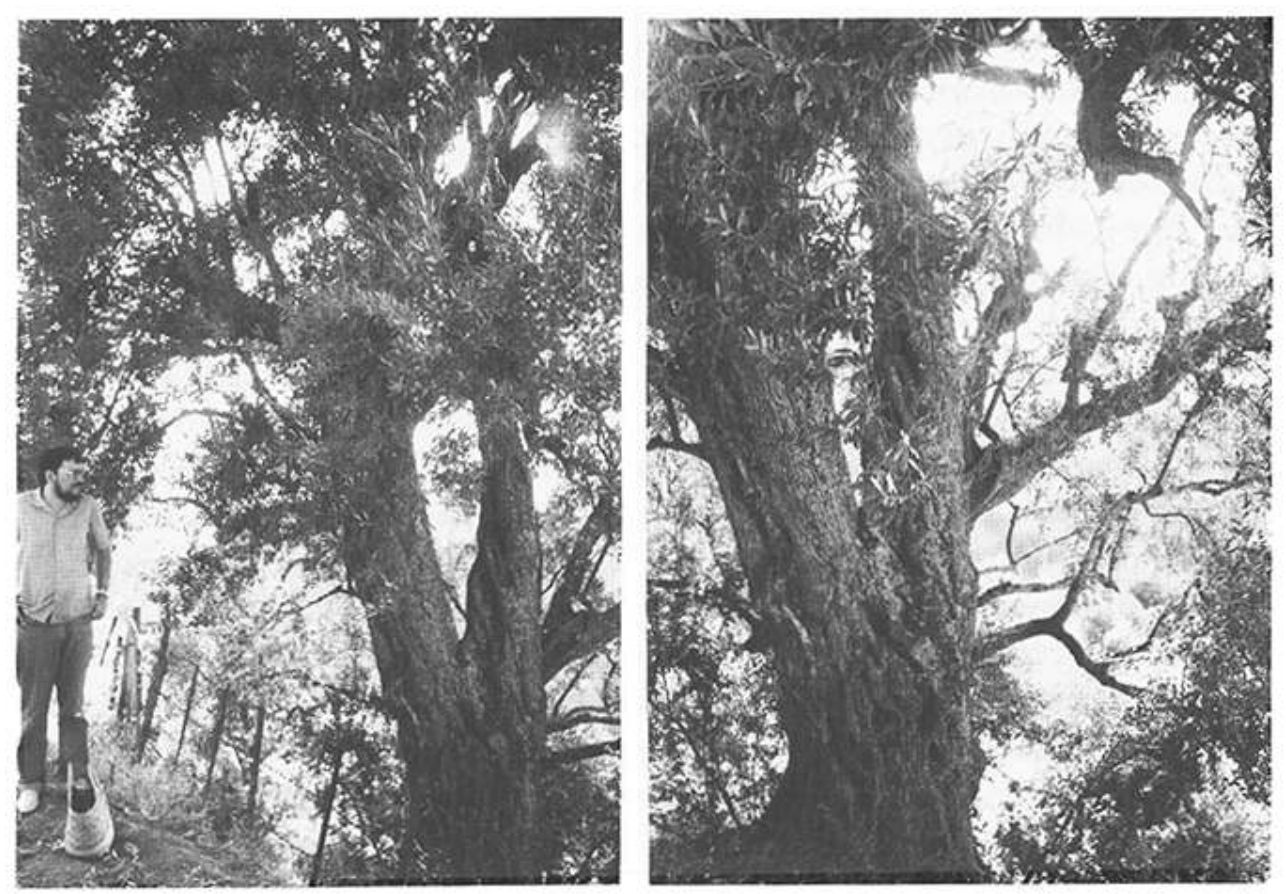

Fig. 1a \& 1b : Un OLIVIER MULTI-CENTENAIRE EN KABYLIE (TAgHANIMT/ADENI, MAISON CHAKER, 1992. CLICHÉ D. CHAKER). EN KABYLIE, LES OLIVIERS PEUVENT ATTEINDRE UNE TAILLE ET UN ÂgE CONSIDÉRABLES.

Et, certes, l'origine latine (ou grecque ?) de āliw, bien que plausible, doit être considérée comme une simple hypothèse - et sans doute pas comme la plus probable. Car le terme

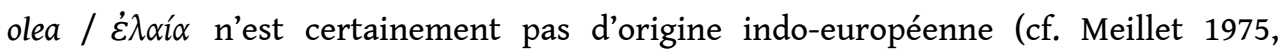
p. 302-303); et, comme l'olivier (sauvage) pousse spontanément sur le pourtour méditerranéen, on doit postuler que les Indo-européens l'ont emprunté à une langue «méditerranéenne » - ou "égéenne » selon l'expression de Meillet - lorsqu'ils sont arrivés sur les rivages de la Méditerranée. Le terme est déjà attesté en grec mycénien ${ }^{3}$, ce qui implique qu'il a été emprunté à une langue "méditerranéenne " à date très ancienne, bien antérieure à l'installation des Grecs en Cyrénaïque $(\mathrm{VII})$ : l'hypothèse d'un emprunt par le grec au berbère dans cette région est donc certainement à écarter. Mais d'autres possibilités restent ouvertes : emprunts parallèles du grec et du berbère à une langue "méditerranéenne» non identifiée, emprunt du grec à une langue «méditerranéenne » qui, elle-même, l'aurait emprunté au berbère, enfin emprunt $d u$ grec archaïque au berbère lors de contacts bien antérieurs à la fondation des colonies de Cyrénaïque... Les données linguistiques, ethno-et paléobotaniques examinées ci-dessous conduisent plutôt à privilégier cette dernière hypothèse.

5 Car un élément important doit être versé au dossier : il n'est pas du tout impossible qu'il existe des traces de āliw (ou des formes apparentées), dans d'autres dialectes berbères, notamment le kabyle. Boulifa (1913, p. 156) avait déjà attiré l'attention dans son petit glossaire sur le nom kabyle d'un arbuste, le filaria (ou filaire: Phillyrea angustifolia L.) de la famille des Oléacées: tamətwala. Le Phillyrea angustifolia évoque l'olivier par son aspect, avec ses feuilles étroites, d'un vert assez sombre, lancéolées, de 2 à $6 \mathrm{~cm}$, et son feuillage persistant. Boulifa proposait d'analyser ce mot comme un composé de tamət + wala, qu'il mettait en relation avec āliw touareg. On considérera ce découpage comme tout à fait recevable et même quasiment certain car il est difficile de voir dans un tel mot une forme simple ou une forme dérivée par préfixe $m$ - sur une 
racine TWL (?), bien improbable. On rappellera par ailleurs que le domaine de la botanique est l'un de ceux où l'on rencontre le plus grand nombre de composés en berbère (cf. Laoust 1920, p. 492-495; pour une approche plus globale de composition lexicale en berbère, voir Chaker 1984, chap. 10). En revanche, son explication du premier élément tamet - qu'il relie au mot tamət/taməțtut, « femme » - doit absolument être rejetée; d'abord pour des raisons phonologiques: le /ț/ de taməț/tamətțut est fondamentalement pharyngalisé ( emphatique »), ce qui n'est pas du tout le cas du /t/ final du premier élément tamət-, mais aussi pour des raisons de vraisemblance sémantique. Il est beaucoup plus simple de considérer ce composant tamət comme un nominal féminin singulier, tout à fait régulier avec sa marque discontinue de genre $t-t$, qui peut être mis en relation avec le touareg tămat qui désigne justement un arbre, le "gommier femelle " ${ }^{4}$. Le second composant, wala, pourrait bien être le correspondant kabyle de āliw, dont le /ā/ initial long et non-alternant à l'Etat d'Annexion* indique la présence d'une ancienne consonne initiale ${ }^{5}$, sans doute la semi-consonne $/ \mathrm{w} /$ ou la laryngale $/ \mathrm{h} /\left({ }^{*}[a]\right.$ wliw $/ *[a]$ hliw), et conforte le rapprochement avec le kabyle -wala et l'hypothèse d'une racine primitive commune *WLW ou ( $\left.{ }^{*} \mathrm{HLW}\right)$. On peut donc, sans difficultés insurmontables, au plan sémantique comme au plan formel, relier le āliw touareg au probable *wala(w) kabyle, impliqué par tamətwala. Il convient aussi de souligner que le mot āliw, āléw/āliwən est parfaitement bien intégré aux structures morphologiques de la langue, par son schème et par son pluriel, ce qui est un indice de son ancienneté, sinon de son « indigénéité ».

6 Si la même racine, désignant des arbustes endémique de la même famille botanique, se retrouve en touareg et en kabyle, l'hypothèse d'une autochtonie de āliw/*WLW n'est plus du tout à exclure ; et par voie de conséquence, celle d'un emprunt, direct ou indirect, du grec au berbère devient une piste sérieuse, alors que l'hypothèse inverse - grec> berbère - devient symétriquement très improbable. On rappellera à ce sujet que les contacts entre le monde grec/égéen et le monde berbère sont très anciens et remontent au moins aux derniers siècles du second millénaire avant J.-C. (cf. Camps 1985, p. 50-51), et sont donc à la fois bien antérieurs à la fondation des colonies de Cyrénaïque (VII ${ }^{e}$ siècle avant $\mathrm{J}$.-C.) et compatibles avec la présence du terme en grec mycénien (XIV $V^{e}$ avant J.-C.). Hérodote lui-même signale expressément plusieurs emprunts technologiques ou culturels faits par les Grecs aux Berbères. Et, si l'on a longtemps eu de fortes réticences à admettre l'emprunt culturel dans ce sens, c'est, comme l'écrit fort justement G. Camps (1985, p. 50), parce que :

«Obnubilés par le génie grec nous admettons difficilement que les Libyens, ces barbares, ces «traînards maghrébins" pour reprendre une expression d'E.-F. Gautier, aient pu enseigner quoi que ce soit aux Hellènes ». 


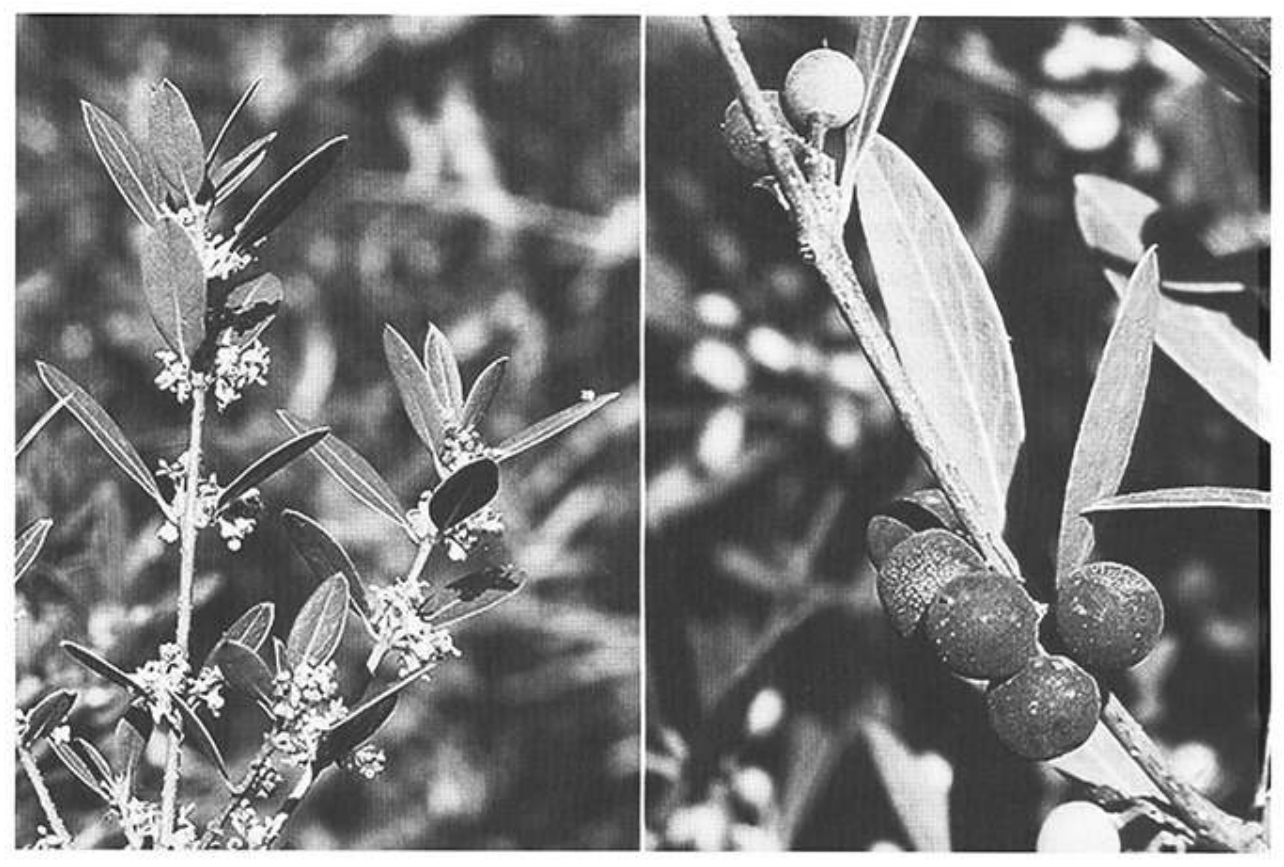

Fig. 2a \& $2 b$ : FILARIA OU filAiRE (PHILLYREA ANgUSTIFOLIA L.) : FEUILLES, FLEURS ET FRUITS

Cliché M. Gast

7 On en vient aussi logiquement à formuler l'idée que āliw, « oléastre » a dû précéder en berbère azəmmur, « olivier/oléastre », qui est vraisemblablement une forme secondaire qualifiante (cf. supra).

8 Bien entendu la question, proprement linguistique, de l'origine du terme aliw ne peut être totalement séparée de celle, historique et ethnologique, de l'origine de la culture/ exploitation de l'olivier en Afrique du Nord. Ce point a été rapidement abordé dans la notice «Huile» (H62, EB XXIII, 2000) et surtout ici même dans les notices botanique (013, par F. Abdoun) et historique (014 par J.-P. Laporte) consacrées à l'olivier. La paléobotanique (notice 013) établit à la fois l'origine africaine du genre Olea et la présence très ancienne du pollen d'Olea europaea en Afrique du Nord: dès le début l'Holocène dans plusieurs sites avec, en Kroumirie* (Tunisie du Nord), une présence continue depuis - 20000 . Pour ce qui est de son exploitation et de sa culture, G. Camps, qui a consacré un passage conséquent à l'olivier dans son Massinissa (1961, p. 87-91), considère que les Berbères pratiquaient la greffe de l'oléastre avant l'influence phénicienne et signale (p. 47 et 87 ) que, selon le témoignage explicite du pseudoScylax ${ }^{6}$, «les habitants de Djerba savaient tirer de l'huile des fruits de l'oléastre ». Autrement dit, les Berbères exploitaient, au milieu du IV ${ }^{e}$ siècle avant J.-C., les fruits de l'arbuste indigène non cultivé, ce qui rend finalement assez improbable l'hypothèse d'un emprunt de sa dénomination au grec. On notera que Laoust (1920, p. 444) mentionne que les Chleuhs du Haut-Atlas extraient encore de l'huile «amère, de peu d'usage, réservée à l'éclairage » du fruit de l'oléastre! Plus largement, il paraît bien établi que dans toute la Méditerranée occidentale, la domestication - à tout le moins une "pré-domestication" - de l'olivier est bien antérieure à l'oléiculture antique, punique ou romaine, et s'enracine dans le Néolithique (Terral 1997). En Afrique du Nord comme dans tout le bassin occidental de la Méditerranée, l'influence punique, romaine, éventuellement grecque, a été tardive et a porté plus certainement sur des aspects agronomiques tels que les techniques et les pratiques de culture (mise en place 
de vergers, taille, irrigation...), des pratiques d'amélioration variétale (introduction de variétés allochtones, hybridations avec des formes locales et sélection) et les techniques de production de l'huile (pressoirs), sans doute aussi de stockage et de commercialisation.

9 Signalons enfin à propos de cette forme touarègue, fort intrigante, que le pluriel âliwən désigne aussi un chant rituel du mariage :

«... L'emploi du rythme āliwən est exclusivement réservé à des vers chantés par les femmes dans certaines cérémonies des noces. Les vers, très peu nombreux et tous très anciens, composés sur ce rythme font partie du cérémonial des noces et se transmettent de génération en génération sans qu'on sache quand ni par qui ils ont été faits. Le rythme āliwən est originaire de l'Ajjer et est très ancien. [...] Les femmes du campement où a lieu le mariage chantent en chœur des vers du rythme àliwən dans la matinée du jour où se fait le mariage. » (Foucauld, III, p. 1094).

10 Il est assez remarquable au plan symbolique que le mariage soit aussi clairement associé à l'olivier : évocation de la durabilité, de la capacité de régénérescence, de la fécondité en référence à la multiplicité des fruits produits... ?

Même s'il existe d'autres termes locaux (par ex., kabyle: aḥeššad), tous les parlers berbères Nord qui ont azəmmur = "olivier " emploient le terme az̧əbbuž (et fem. taz̧əbbužt) pour désigner l'oléastre (Laoust 1920, p. 444). Le terme est ancien et déjà donné par une source arabe au XI ${ }^{e}$ siècle (Al-Išbilī, cf. Bustamante, Corriente et Tilmatine 2007, vol. II, p. 342, $\mathrm{n}^{\circ} 2231$ ). Il se rencontre aussi en arabe dialectal (zəbbuž, žabbuž, zənbuž... ; cf. Colin 1927, 1993/7 ; de Prémare 1993/9), y compris en maltais (Barbera 1940, IV). Des formes très voisines sont connues en espagnol (andalous: zebuche; castillan : acebuche...) et en portugais (zambujeiro, azambujeiro). Le dialectologue Georges S. Colin (1927, p. 89-91) a abordé très précisément la question de l'origine et de la distribution de ce terme "arabo-berbère "; pour lui le mot n'est ni berbère ni sémitique et il reprend à son compte l'hypothèse d'une origine latine, formulée depuis longtemps par les romanistes (Meyer-Lübke, Simonet...) : acerbus, «amer, âpre » ou *acifolium < acrifolium (" arbre à feuillage amer») ou aquifolium (" arbre à feuillage piquant»). Al-Išbīlī (XI siècle) signale que le fruit de l'oléastre s'appelle zaǵbağ (Bustamante, Corriente et Tilmatine 2007, $\mathrm{n}^{\circ}$ 2231), ce qui laisse ouverte la possibilité d'une autre étymologie et d'une autre origine. Le Dictionnaire des racines sémitiques (Cohen et al., fasc. 8, 1999, p. 670) évoque, prudemment, la possibilité d'un lien avec une racine ZBG, «noir, jais... ». S'appuyant sur la distribution géographique très large du mot - toute la péninsule Ibérique et tout le Nord de l'Afrique -, Camps (1961, p. 89) a émis l'hypothèse qu'il pourrait s'agir d'un terme très ancien, probablement prélatin, dont l'aire d'extension s'expliquerait par une diffusion secondaire par le latin. Au plan formel, si sa structure morphologique correspond à un schème courant en berbère - le même que azəmmur d'ailleurs -, sur le plan phonotactique elle peut difficilement être considérée comme une séquence radicale berbère acceptable: la présence concomitante dans la même racine de la dentale constrictive /z/ et de la prépalatale constrictive /ž/ en trahit de façon immédiate une origine étrangère ou expressive, ce qui tend à corroborer la position de Colin (1927). On peut, en conséquence, penser que le terme berbère primitif pour désigner l'oléastre était āliw, attesté en touareg et sans doute en kabyle, et que azəabbuž est une dénomination secondaire, un " mot voyageur » diffusé par les Latins. 
12 S'il existe bien un nom indigène de l'olivier (azəmmur) et de l'oléastre (āliw), curieusement, le nom de l'huile en revanche a été emprunté, à deux reprises à des langues sémitiques, le punique puis l'arabe :

13 - La quasi-totalité des dialectes berbères actuels, en dehors du touareg, a la forme zzit (et variantes phonétiques), manifestement empruntée à l'arabe (dialectal: (z)zit; classique : zayt). Contrairement à ce que semble penser Camps (1961, p. 87), aucun indice linguistique ne permet de penser que le terme est d'origine punique : la racine est évidemment la même en punique et en arabe (ZYT), mais la vocalisation et la morphologie (article défini initial) en sont différentes et celles relevées en berbère sont bien celles de l'arabe dialectal (/zz/ tendu initial résultant de l'assimilation automatique de l'article défini arabe al- et voyelle /î/). Aucun terme berbère n'a jamais été relevé dans aucun dialecte Nord, alors qu'il existe plusieurs mots largement répandus pour désigner les graisses d'origine animale : udi, «beurre ", talussi, "beurre frais, crème ", tadunt, "graisse »... Et, contrairement à ce qu'affirme Laoust (1920, p. 446) sans arguments probants ${ }^{7}$, il ne paraît pas possible d'attribuer à udi la signification première de " huile » : tous les grands dialectes (kabyle, chleuh, touareg, rifain, tamazight...), très éloignés géographiquement les uns des autres, lui donnent le sens de «beurre (frais/ salé) » et seul le groupe des parlers « orientaux » (Tunisie-Libye) a « huile » (cf. NaïtZerrad, II, 1999, p. 275).

14 Situation très paradoxale vu l'importance et l'ancienneté de la culture de l'olivier et des technologies de la production de l'huile en Afrique du Nord, bien antérieurement à l'arrivée des Arabes. Une explication possible de cette intrusion massive de l'arabe dans ce champ lexico-sémantique pourrait être que, très tôt, des Arabes dans les grandes régions oléicoles (Tunisie et Tripolitaine notamment, mais aussi en Andalousie) ont pris le contrôle du commerce de l'huile, sans doute économiquement stratégique.

15 - Le touareg (tamahĂq de l'Ahaggar-Ajjer), sur ce point aussi, se distingue nettement du berbère Nord: il présente des formes ahātim «olive, huile d'olive» / fem. təhātimt, " olivier cultivé » (Foucauld, II, 679-680 \& Laoust 444-445); ahātim repose sur une forme première azātim, confirmée par azātšim attesté à Ghat (Nehlil 1909, p. 168), le /h/ de la tamahăq provenant très souvent de /z/ (Prasse 1969). Ce terme est lui-aussi un emprunt au sémitique, mais en l'occurrence au punique, comme l'indique immédiatement le suffixe de pluriel caractéristique -im. L'étymon punique étant un pluriel à valeur de collectif : zêt-ìm ; « huiles » (cf. Vycichl 1952, p. 201).

16 Ce qui confirme que les Puniques ont aussi joué un rôle important dans l'exploitation de l'olivier.

17 - L'olive, dans les dialectes qui ont azəmmur = «olivier cultivé », est normalement

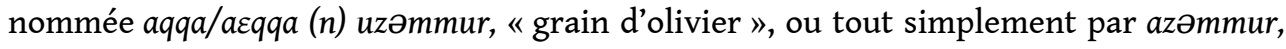
qui a valeur de collectif et désigne à la fois l'espèce et ses fruits: пәс̌čA azəmmur = « nous avons mange des olives ». Les autres parlers, ceux qui ont azəmmur = « oléastre » et ceux qui ne connaissent pas du tout azəmmur, empruntent le terme arabe zzitun, zzitunət, ou la forme berbérisée tazitunt, pour désigner le fruit ou emploient divers syntagmes dont le centre est zzitun (chleuh : tagəmmut n zzitun, " grain d'olivier »).

18 En touareg, c'est ahātim qui est employé à la fois pour désigné l'huile d'olive et l'olive (de l'oléastre, l'olivier cultivé n'étant pas connu).

19 On terminera cet examen des matériaux linguistiques en reprenant - mais en la nuançant sérieusement - la conclusion d'Emile Laoust (1920, p. 448) : 
«Ces constatations d'ordre linguistique concordent parfaitement avec les données historiques qui nous apprennent que les Phéniciens, les Grecs, les Romains et enfin les Arabes se sont intéressés, à des titres divers, à la culture, et à l'exploitation du précieux végétal. »

20 Mais les deux dénominations fondamentales de l'oléastre et de l'olivier (āliw / azəmmur), elles, sont très certainement autochtones et antérieures à toute influence étrangère.

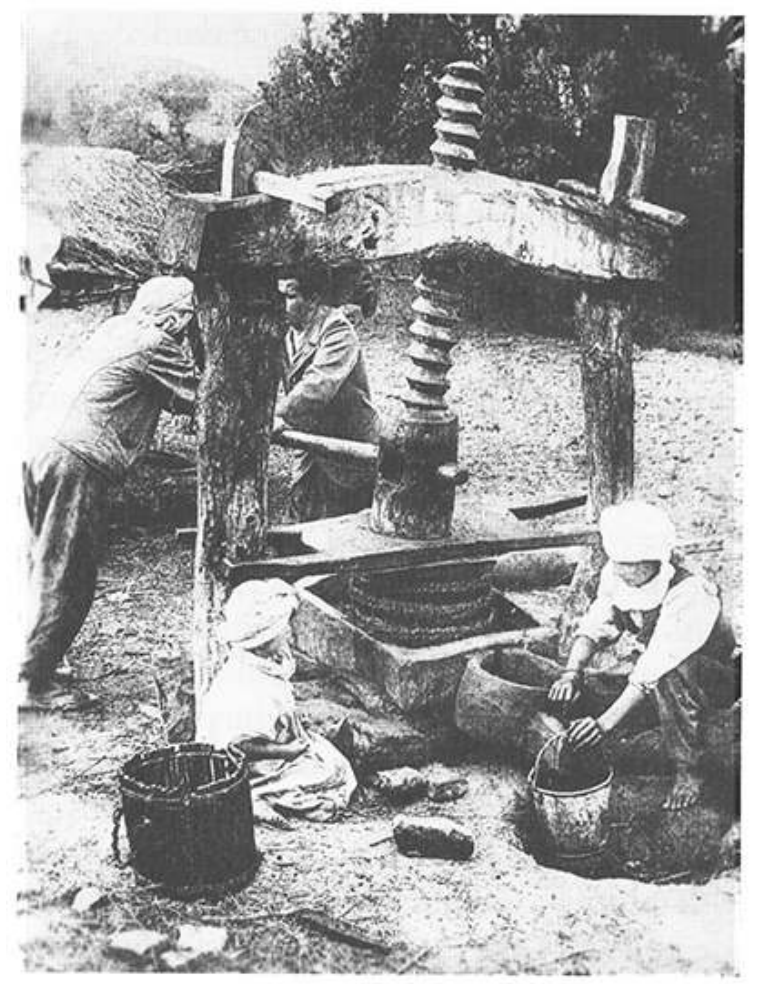

FIg. 3 : PRESSOIR À OLIVES À VIS EN ACTION.

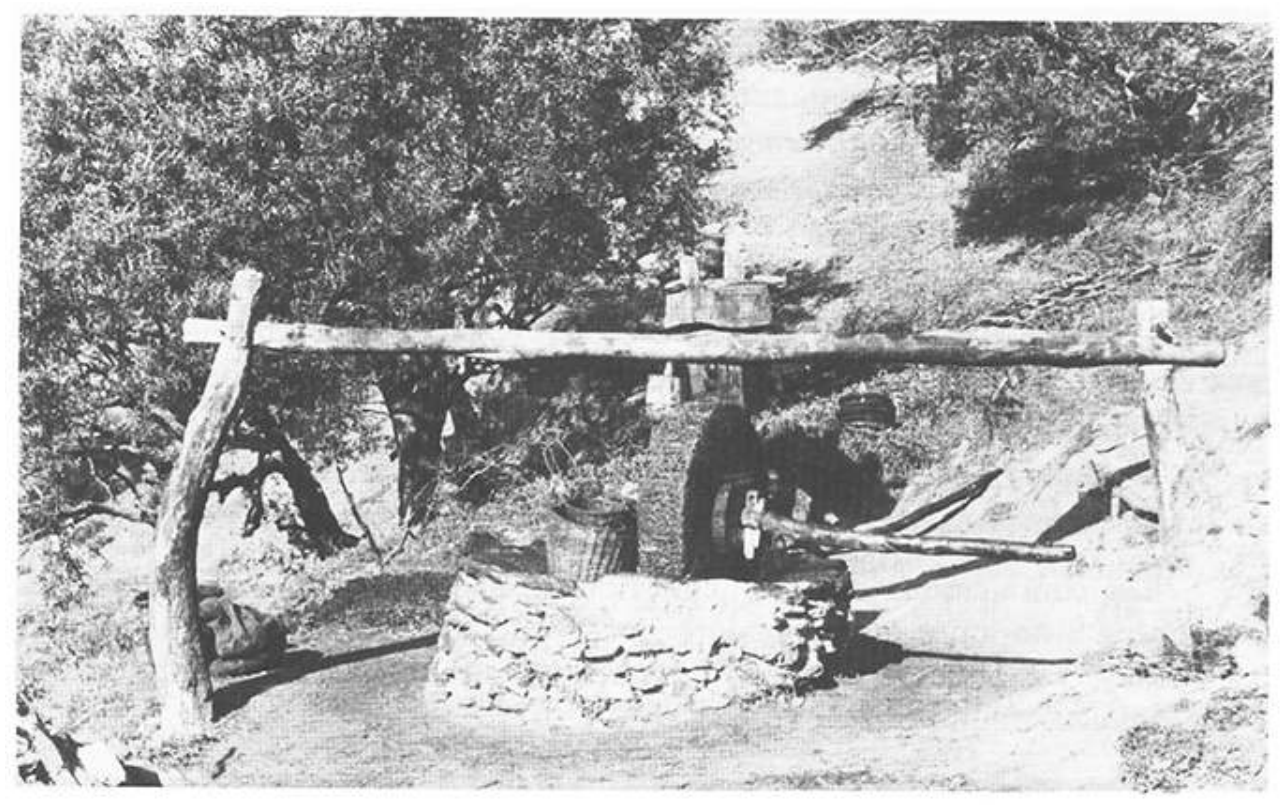

Fig. 4 : Meule à olives. (Kabylie, Aït Ittsouragh, 1940. Clichés J. Lanfry - Collection du Centre de Recherche Berbère de l'INALCO). 
$21 \rightarrow$ Voir aussi "Aliw (olivier sauvage)" (A167), EB IV, 1987 (S. Chaker); “Azemmur (olivier)" (A346), EB VIII, 1990 (S. Chaker); “Huile” (H62), EB XXIII, 2000 (H. CampsFabrer).

\section{BIBLIOGRAPHIE}

AG SIDIYENE E., Des arbres et des arbustes spontanés de l'Adrar des Ifoghas (Mali). Etude ethnolinguistique et ethnobotanique, Paris, ORSTOM/CIRAD, 1996.

BARBERA G., Dizionario maltese-arabo-italiano. Con una grammatica comparata arabo-maltese, 4 vol., 1939/1940, Imprimerie Catholique du Liban.

BASSET A., La langue berbère, Londres, 1952 (1969).

BEGUINOT F., Il Berbero Nefûsi di Fassâto, Roma, 1931, p. 263.

BESNARD G., RUBIO DE CASAS R., CHRISTIN P. A., VARGAS P., "Phylogenetics of Olea (Oleaceae) based on plastid and nuclear ribosomal DNA sequences : Tertiary climatic shifts and lineage differentiation times", Annals of Botany, 104, 2009, p. 143-160.

BRockelmanN C., Précis de linguistique sémitique (trad. W. Marçais et M. Cohen), Paris, Geuthner, 1910.

BOULIFA A., Lexique kabyle-français, Glossaire, Alger, Jourdan, 1913.

BUSTAMANTE J., F. CORRIENTE F. y M. TILMATINE M. (Eds.), AbulHayr Al-'Išbīlīi, Kitāau 'Umdati ț-țabib fï ma'rifati nnabāt likulli labīb (Libro base del médico para el conocimiento de la Botánica por todo experto) / s. V/XI), edición, notas y traducción castellana, Madrid, 2007, Consejo Superior de Investigaciones Científicas, vol. II (Fuentes arábico-hispanas : 33).

CAMPS G., Massinissa, ou les débuts de l'histoire, 1961, Alger, Imprimerie officielle.

CAMPS G., « Pour une lecture naïve d'Hérodote. Les récits libyens (IV, 168-199) », Storia della Storografia, 1985, 7, p. 38-59.

CHAKER S., Textes en linguistique berbère (Introduction au domaine berbère), Paris, Editions du CNRS, 1984.

CHANTRAINE P., Dictionnaire étymologique de la langue grecque : histoire des mots, 1968-1980 (nouvelle édition 2009), Paris, Klincksieck.

COHEN D. et al., Dictionnaire des racines sémitiques..., Louvain/Paris, Peeters, 1993-2011 (10 fasc. parus).

COLIN G. S., « Etymologies maġribines », Hespéris, VII, 1927, p. 89-91.

COLIN G. S., Le dictionnaire COLIN d'arabe dialectal marocain (sous la dir. de Z. Iraqui-Sinaceur), Rabat, Éditions Al-Manahil/Ministère des Affaires Culturelles, 1993-7, 8 vol.

DALLET J.-M., Dictionnaire kabyle-français, Paris, 1982, p. 948.

DESTAING E., Vocabulaire français-berbère (tachelhit du Sous), Paris, 1920, p. 204. 
DESTAING E., Vocabulaire français-berbère (dialecte des Beni-Snous), Paris, 1914, p. 252.

ERNOUT A. \& MEILLET A., Dictionnaire étymologique de la langue latine, Paris, Paris, Klincksiek, 1994

(1 $1^{\text {ère }}$ éd. : 1922).

ESTEBAN IBANEZ Fr., Diccionario español-rifeño, Madrid, 1944, p. 305.

FOUCAULD Ch. de, Dictionnaire touareg-français (dialecte de l'Ahaggar), Paris, Imprimerie Nationale, 1950-1952, 4 tomes.

GREEN P. S., “A revision of Olea L.”, Kew Bulletin, 57, 2002, p. 91-140.

HUYGHE R.P., Dictionnaire français-chaoui, 1906, p. 455.

LAOUST E., Mots et choses berbères, Paris, 1920, p. 444 et sq.

MEILLET A., Linguistique historique et linguistique générale, Paris, Champion, 1975 : «A propos du nom du vin et de l'huile ", p. 297-304.

MERCIER H., Vocabulaire et textes berbères (Ait Izdeg), Rabat, 1937, p. 180.

NAÏT-ZERRAD K., Dictionnaire des racines berbères..., II, Paris/Louvain, Peeters, 1999.

NEHLIL, M., Étude sur le dialecte de Ghat, Paris, E. Leroux, 1909.

OULEBSIR R., L'olivier en Kabylie entre mythes et réalités, Paris, L'Harmattan, 2008.

PRASSE K.-G., A propos de l'origine du H touareg (Tahaggart), Copenhague, Munksgaard, 1969.

PRASSE K.-G., Manuel de grammaire touarègue (TĂhĂggart), IV-V, Nom, Copenhague, Akademisk Forlag, 1974.

PRASSE K.-G. et al., Dictionnaire touareg-français, Copenhague, Museum Tusculanum Press/ University of Copenhaguen, 2003.

PREMARE (de) L., 1993/1999 - Dictionnaire arabe-français, Langue et culture marocaines, Paris, L'Harmattan (12 vol.).

QUEZEL \& SANTA, Nouvelle flore d'Algérie et des régions désertiques méridionales, Paris, Éditions du CNRS, 1962/1963, t. 1 \& 2 .

RICARD R., « Latin "olea”, touareg et portugais "aléo", hypothèses et rapprochements ", Bulletin hispanique, t. 63/3-4, 1961, p. 179-185.

TERRAL J.-F., 1997 - La domestication de l'olivier (Olea europaea L.) en Méditerranée nord-occidentale : Approche morphométrique et implications paléoclimatiques, Thèse de Doctorat, Université Montpellier-II.

VYCICHL W., "Punischer Spracheinfluss im Berberischen", Journal of Near eastern Stdues, XI/3, 1952, p. 198-204.

VYCICHL W., “Berberisch Z-M-R “KÖNNEN”, “POTERE””, Rivista degli Studi Orientali, XXXVII, 1962, p. 77-78.

\section{NOTES}

1. Schème de formation de nominaux très fréquent dans le lexique berbère (ex.: akəmmus «ballot, paquet, nouet» < kməs, " emballer, empaqueter, enserrer, amasser »...), qui a des pendants dans certaines langues sémitiques (cf. Brockelmann 1910, p. 158 ; aussi Prasse 1974) : il 
s'agit d'un participe passif (ou "adjectif verbal") en /u/ sur thème verbal d'intensif (à tension de la seconde radicale).

2. Olea europaea subsp. laperrinei selon Green (2002) ; Olea laperrinei Batt. \& Trab., nous précise M. Thinon.

3. Information de Jean-Victor Vernhes, hélléniste et comparatiste de l'Université d'Aix-Marseille. Le grec mycénien (linéaire B) est daté du XIV e siècle avant J.-C.

4. Acacia seyal Del. selon Foucauld, III, p. 1139) ou Quézel \& Santa (1963). M. Thinon nous signale qu'il s'agit en fait de : Acacia ehrenbergiana Hayne.

5. Rappelons que les berbérisants considèrent depuis longtemps (cf. notamment Basset 1952, p. 27-28) que la voyelle initiale stable des noms (i.e. maintenue à l'Etat d'Annexion, contrairement à la règle) est l'indice de la disparition d'une ancienne consonne radicale initiale ; par ex. : tala, «fontaine » (kabyle) est un nom à voyelle non-alternante, alors que son correspondant touareg tahala, « source » connaît l'alternance régulière [a/ə].

6. "Ils font beaucoup d'huile avec le fruit de l'olive sauvage ", Pseudo-Scylax, Périple, 110 (texte et trad. de E. Cougny \& H. Lebègue, Paris, Renouard, 1878-1892 ; consulté sur Internet).

7. Tous les rapprochements formels et sémantiques qu'il opère (au bas de la p. 444 et p. 446), autour d'une prétendue racine $D$, sont très fragiles et doivent être rejetés; udi, qui repose sur une racine *WDY, n'a certainement rien à voir avec tadunt, "graisse" (qui renvoie à une racine chamito-sémitique DHN), ni avec $n d w . .$. «baratter » (qui réfère très certainement au mouvement de l'outre-baratte ; cf. $n d w$, « sauter, bondir, traverser... »), ni avec les autres formes qu'évoque Laoust.

\section{INDEX}

Mots-clés : Agriculture, Antiquité, Botanique, Ethnographie, Kabylie, Littérature 